\title{
Nachruf auf Prof. Dr. Dr. h.c. mult. Karl Martin Bolte
}

Online publiziert: 7. April 2011

(C) Institut für Arbeitsmarkt- und Berufsforschung 2011

Mit Karl Martin Bolte verstarb einer der einflussreichsten Nachkriegssoziologen Deutschlands. Er war von 1970 bis 1999 prägender Gründungs- und Mitherausgeber der Mitteilungen aus der Arbeitsmarkt- und Berufsforschung, aus denen 2004 die ZAF hervorging. Auch am Aufbau des IAB hatte Bolte in beratender Funktion maßgeblichen Anteil. So war er schon in den Jahren 1967 bis 1972 Mitglied des wissenschaftlichen Beirats (später: Sachverständigengremium) des IAB, welches die Gründung des Instituts und dessen Arbeit während der ersten Jahre begleitete.

Es sind Namen wie Karl Martin Bolte, die einem zuerst in den Sinn kommen, wenn es um den Aufbau und institutionellen Ausbau der Soziologie nach 1945 geht. Boltes Arbeiten zur Sozialstrukturanalyse und zur Arbeits- und Berufssoziologie waren ebenso wegweisend wie seine nachhaltigen und erfolgreichen Bemühungen, das Verhältnis zwischen Theorie und Praxis wie auch das zwischen Theorie und Politik zu verbessern. Wissenschaft und Praxis waren für Bolte nach eigenem Bekunden ,gleichrangige Partner, die es in ihrer Arbeit weit unmittelbarer und intensiver zu verflechten gilt“". Daher verwundert es auch nicht, dass Bolte der Überzeugung war, dass mit der Sprache eines gebildeten Nicht-Wissenschaftlers im Prinzip alles ausgedrückt werden könne, was ein Sozialwissenschaftler zu sagen habe.

Blickt man auf Boltes wissenschaftliches Wirken, so zählt seine Analyse der Sozialstruktur der Bundesrepublik Deutschland, insbesondere mit seiner eingängigen Darstellung des gesellschaftlichen Schichtungsgefüges in Form ei- ner Zwiebel, gewiss zu seinen bekanntesten Erfolgen. Denn bis heute gibt es wohl kaum eine sozialwissenschaftliche Abbildung, die häufiger abgedruckt wurde als ebendiese von ihm in den sechziger Jahren entwickelte Zwiebel. Auch wies Bolte schon in den siebziger Jahren auf die gravierenden sozialen und politischen Folgen des demographischen Wandels hin. Mit der von ihm maßgeblich geprägten ,subjektorientierten Soziologie“ rückte er die handelnden Personen ins Zentrum der theoretischen Betrachtung und der empirischen Forschung. Dem Konstruktivismus eines Niklas Luhmann stand er skeptisch gegenüber. Wer im Krieg die Gewalt der Dinge erlebt habe, so Friedhelm Neidhardt in einer Laudatio zu Boltes achtzigstem Geburtstag, tue sich schwer mit dem Glauben, man bilde sich alles nur ein. Gleichwohl war ihm die Pluralität wissenschaftlicher Perspektiven eine Selbstverständlichkeit, akademischer Widerspruch willkommen.

Mit Blick auf die Arbeitsmarktforschung waren insbesondere Boltes vielfältige Arbeiten auf dem Feld der soziologischen Bildungs- und Berufsforschung prägend. Davon zeugen nicht zuletzt seine stark rezipierten Veröffentlichungen in den „Mitteilungen aus der Arbeitsmarkt- und Berufsforschung“", der Vorgängerzeitschrift der ZAF (siehe unten).

Schließlich hat Bolte sich auf dem Feld der politischen Bildung bleibende Verdienste erworben, nicht zuletzt bei der Einführung der Sozialkunde als ordentliches Schulfach in Deutschland.

Karl Martin Bolte verstarb am 14. Februar 2011 im Alter von 85 Jahren. 
Veröffentlichungen von Karl Martin Bolte in den ,Mitteilungen aus der Arbeitsmarkt- und Berufsforschung““

Bolte, K.M. (1969): Untersuchung über Ergebnisse der beruflichen Fortbildung im Rahmen des „Individuellen Förderungsprogramms“ Eine Befragung bei den im Jahre 1964 Geförderten. In: Mitteilungen des Instituts für Arbeitsmarkt- und Berufsforschung, 2. Jg., Heft 07, S. 509-520

Bolte, K.M. (1971): Wissenschaft und Praxis - Möglichkeiten ihres Verhältnisses zueinander. In: Mitteilungen aus der Arbeitsmarkt- und Berufsforschung, 4. J., Heft 4, S. 356-365

Beck, U., Bolte, K. M., Brater, M. (1976): Bildungsreform und Berufsreform. Zur Problematik der berufsorientierten Gliederung des Bildungssystems. In: Mitteilungen aus der Arbeitsmarkt- und Berufsforschung, Jg. 09, Heft 4, S. 567-674

Bolte, K.M. (1991): Das gesellschaftliche Umfeld von Arbeitsorganisationen als Gegenstand beruflicher Weiterbildung. In: Mitteilungen aus der Arbeitsmarkt- und Berufsforschung, Jg. 24, Heft 2, S. 263-269

Bolte, K.M. (1997): Zur Innovation eines wissenschaftlichen Ansatzes Arbeitsmarkt- und Berufsforschung in der Wissenschaftslandschaft und Politikberatung. In: Mitteilungen aus der Arbeitsmarkt- und Berufsforschung, Jg. 30, H. 4, S. 801-806 Kostenlose Downloads der Volltexte ab 1971 unter www.iab.de/mitteilungen

\section{Karl Martin Bolte}

* 29. November 1925 in Wernigerode/Harz

$\dagger$ 14. Februar 2011 in Gauting

\section{Schule}

Gymnasium in Wernigerode

1946 Abitur

\section{Ausbildung}

1947-1950 Studium der Wirtschafts- und Sozialwissenschaften an der Christian-Albrechts-Universität Kiel

1950 Diplomvolkswirt an der Rechts- und Staatswissenschaftlichen Fakultät der Universität Kiel

1952 Promotion zum Dr. rer. pol. an der Universität Kiel

1957 Habilitation für Soziologie an der Universität Kiel

\section{Beruflicher Werdegang}

1950-1957 Assistent bei Gerhard Mackenroth

Seit 1952 Dozententätigkeit im Bereich sozialwissenschaftlich ausgerichteter Erwachsenenbildung

1957-1961 Universitätsdozent an der Universität Kiel

1961-1964 Professor für Soziologie an der Hochschule für Wirtschaft und Politik in Hamburg

1962-1964 Leiter der Hochschule für Wirtschaft und Politik und Honorarprofessor an der Universität Hamburg

1964-1992 Professor für Soziologie an der Ludwig-Maximilians-Universität München, Vorstand des Instituts für Soziologie

\section{Sonstige Funktionen und Ämter}

1967-1972 Mitglied des Sachverständigengremiums des Instituts für Arbeitsmarkt- und Berufsforschung der Bundesanstalt für Arbeit

1969-1971 Vorsitzender der Kommission zur Erstellung des 3. Jugendberichts

1970-1999 Mitherausgeber der Mitteilungen aus der Arbeitsmarkt- und Berufsforschung

1971-1976 Vorsitzender der Kommission „Wirtschaftlicher und sozialer Wandel der Bundesrepublik Deutschland“

1972-1986 Sprecher des Sonderforschungsbereichs 111 „Theoretische Grundlagen sozialwissenschaftlicher Arbeitskraft- und Berufsforschung" der Ludwig-Maximilians-Universität München

1974-1978 Vorsitzender der Deutschen Gesellschaft für Soziologie

1978-1980 Vorsitzender des Arbeitskreises „Geburtenrückgang und nachwachsende Generation“ der Gesellschaft für sozialen Fortschritt

1980-1986 Mitglied des Senats und des Hauptausschusses der Deutschen Forschungsgemeinschaft

1991 Ehrendoktorwürde der Katholischen Universität Eichstätt-Ingolstadt

1993-1999 Beirats- bzw. Kuratoriumsvorsitzender der Schader-Stiftung

2004 Preis der Deutschen Gesellschaft für Soziologie für sein herausragendes wissenschaftliches Lebenswerk 